\title{
Generation of specific antisera directed against D-amino acids: focus on the neuroanatomical distribution of D-glutamate and other D-amino acids
}

\author{
Rafael Coveñas ${ }^{1}$, Arturo Mangas ${ }^{1,2,3}$, Manuel Lisardo Sánchez ${ }^{1}$, Diana Cadena ${ }^{4}$, \\ Marianne Husson', Michel Geffard ${ }^{2,3}$
}

\author{
${ }^{1}$ Institute of Neurosciences of Castilla y León (INCYL), Laboratory of Neuroanatomy \\ of the Peptidergic Systems (Lab. 14), University of Salamanca, Salamanca, Spain \\ ${ }^{2}$ GEMAC S.A., Immunochemistry \& Research Department, Saint Jean d'Illac, France \\ ${ }^{3}$ Institut pour le Développement de la Recherche en Pathologie Humaine et Thérapeutique \\ (IDRPHT), Talence, France \\ ${ }^{4}$ Université François Rabelais, UMR CNRS 7292, Tours, France
}

\begin{abstract}
This review updates the findings about the anatomical distribution (using immunohistochemical techniques) and possible functions of D-glutamate in the central nervous system of mammals, as well as compares the distribution of D-glutamate with the distribution of the most studied D-amino acids: D-serine and D-aspartate. The protocol used to obtain highly specific antisera directed against D-amino acids is also reported. Immunoreactivity for D-glutamate was found in dendrites and cell bodies, but not in nerve fibers. Perikarya containing D-glutamate were found in the mesencephalon and thalamus. The highest density of cell bodies was found in the dorsal raphe nucleus, the mesencephalic central grey matter, the superior colliculus, and in the subparafascicular thalamic nucleus. In comparison with the distribution of immunoreactive cell bodies containing D-serine or D-aspartate, the distribution of D-glutamate-immunoreactive perikarya is less widespread. Currently, the physiological actions mediated by D-glutamate in the brain are unknown but the restricted neuroanatomical distribution of this D-amino acid suggests that D-glutamate could be involved in very specific physiological mechanisms. In this sense, the possible functional roles of D-glutamate are discussed. (Folia Histochemica et Cytobiologica 2017, Vol. 55, No. 4, 177-189)
\end{abstract}

Key words: antisera; D-aspartate; D-glutamate; D-glutamic acid; D-serine; IHC; brain; rat; mapping

\section{Introduction}

Most amino acids have stereoisomers (L- and D-forms) caused by the chiral center on the alpha carbon. In the past, it was assumed that in eukaryotes only the L-series of amino acids could be found, but currently and thanks to advances in analytical techniques, the

Correspondence address: A. Mangas, Ph.D., M.Sc. University of Salamanca, Institute of Neurosciences of Castilla y León (INCYL), Laboratory of Neuroanatomy of the Peptidergic Systems (Lab. 14)

c/ Pintor Fernando Gallego, 1, 37007-Salamanca, Spain

e-mail: mangasam@usal.es enantiomers of L-amino acids, called D-amino acids, have been also found in mammalian tissues, including humans [1-6]. These D-amino acids are present in their free form or in proteins as D-amino acid residues $[1,3]$. In mammals, D-amino acids have been located in the central nervous system (CNS), in peripheral tissues and in physiological fluids [3,7]. The presence of these substances in physiological fluids is influenced by diet, physiological state and age [3].

It has been reported that several synthetic and natural peptides containing D-amino acids showed an antimicrobial activity; these peptides promoted the death of cells by the disruption of bacterial membranes [3]. 
Free D-amino acids (e.g., D-serine, D-aspartate, D-glutamate) are novel physiological substances playing important roles in the mammalian central nervous system, such as synaptic transmission, neuroplasticity, neuronal migration, and brain disorders $[1,2,8-10]$. For instance, D-serine is an endogenous neuromodulator, since it plays a crucial role in N-methyl-D-aspartate (NMDA) receptor-mediated neurotransmission, responsible for memory, learning and behavior [1,3]. In fact, D-serine acts as a co-agonist of the NMDA receptor in the brain of mammals [3]. It is also known that D-serine inhibits the symptoms of the schizophrenia induced by phencyclidine [11], and that treatment of schizophrenic patients with D-serine, as an add-on agent to other antipsychotics, improves negative, positive and cognitive symptoms [12]. D-serine has been also involved in the neuropathogenesis of HIV-1 associated neurocognitive disorders, epilepsy, Alzheimer's disease, amyotrophic lateral sclerosis, neurotoxicity, ischemia and synaptic plasticity, and it has been reported that the extracellular level of D-serine was regulated by the AMPA glutamate receptor [3,13-19]. Moreover, it was found that the level of D-serine decreases with age in the mouse cerebellum [3].

D-aspartate inhibits melatonin synthesis in the pineal gland and stimulates the expression of vasopressin and oxytocin mRNAs, and it was shown that the significant amount of D-aspartate observed in human and rat embryonic brains decreases rapidly after birth [3, 20-22]. D-aspartate has been located in synaptic vesicles and it seems that this D-amino acid is synthesized by the testes and the pituitary gland [3]. D-aspartate has been involved in the development of the nervous system and in neuroendocrine activities (e.g., D-aspartate stimulates the release of luteinizing hormone, gonadotropin-releasing hormone, growth hormone, prolactin, progesterone and testosterone) [3]. It was reported that $\mathrm{D}$-aspartate drinking solution alleviated pain in neuropathic mice [23] and that this D-amino acid is involved in brain aging and spermatogenesis $[3,24]$. In fact, it is known that age-related changes in $\mathrm{D}$-aspartate oxidase promoter methylation regulated the extracellular level of D-aspartate, preventing cell death during brain aging [25]. D-aspartate has been implicated in the regulation of steroid synthesis in the brain [26] and in schizophrenia [24, 27, 28].

D-glutamate is a potent natural inhibitor of glutathione synthesis. In humans, after the oral administration of D-glutamate, the concentration of this D-amino acid is regulated by two mechanisms: 1) transport into cells and its metabolic conversion to D-pyrrolidone carboxylic acid and excretion and 2) the enhancement of D-glutamate clearance by the kidneys [29]. Moreover, D-glutamate is a low-affinity NMDA receptor agonist [30] and it was shown that repetitive stimulation of cultured hippocampal neurons loaded with D-glutamate caused a dramatic shortening of both the rising and decaying phases of NMDA receptor excitatory postsynaptic currents (EPSCs) evoked by autaptic stimulation [30]. These EPSC time courses are mimicked by NMDA receptor currents evoked in outside-out patches by application of D-glutamate. This means that D-glutamate is released as a false transmitter [30].

The relationship between pathophysiological processes and the brain content of D-amino acids has been also increasingly recognized: $\mathrm{D}$-amino acids are present at significantly higher levels in the cerebrospinal fluid (CSF) of patients suffering from Alzheimer's disease, but this finding was not observed in the CSF of patients affected by multiple sclerosis $[1,31]$. In an experimental model of Alzheimer's disease it has been reported that, compared with control rats, the levels of D-leucine, D-alanine, D-proline and D-serine, in hippocampus and cerebral cortex were significantly decreased [15].

\section{The generation of D-amino acids and methods of their detection in tissues and body fluids}

In most cases, $\mathrm{D}$-amino acids are produced by racemization from their corresponding enantiomer antipodes by the action of racemases and for this reason amino acid racemases play a central role in D-amino acid metabolism. The conversion of L-amino acids to D-amino acids occurs because these racemases change the stereochemistry of the chiral alpha-carbon in amino acids [3]. Most amino acid racemases require pyridoxal 5'-phosphate as a coenzyme, but several other require no coenzymes [32]. It should be noted that many reports indicate that exogenous $\mathrm{D}$-amino acids, derived from food and bacterial cell walls, could be distributed in mammalian tissues [1]. D-amino acids are degraded by the flavoenzyme $\mathrm{D}$-amino acid oxidase through a process of oxidative deamination, and the D-amino acid oxidase regulation of D-amino acid levels has been associated with hormone secretion, synaptic transmission, and cognition [3]. In fact, $\mathrm{D}$-amino acid oxidase activity is increased in patients with schizophrenia compared with healthy ones [33].

In general, the amount of $\mathrm{D}$-amino acids in tissues is quite low and hence sensitive and selective methods such as high-performance liquid chromatography, gas chromatography, high-performance capillary electrophoresis, and enzymatic methods are required for their detection and measurements [15, 34-39]. 
Thus, using these methods, the presence of D-alanine, D-proline, D-leucine, D-phenylalanine, D-glutamine, D-tyrosine, D-tryptophan, D-Lysine, D-threonine and D-valine has been reported in mammalian tissues and physiological fluids (e.g., brain, liver, kidney, pituitary gland, blood, urine) [1]. In addition, thanks to the development of highly specific antibodies against D-amino acids, the anatomical distribution of some D-amino acids (D-serine, D-glutamate and D-aspartate) has been widely reported in the mammalian CNS [1, 2, 8, 40-45]. The presence of other D-amino acids such as D-tryptophan, D-cysteine, D-tyrosine or D-methionine was studied in rat brain, but no immunoreactive structures containing these $\mathrm{D}$-amino acids were observed [44].

All the foregoing shows that there are many data in the literature about the presence in tissues of $\mathrm{D}$-serine and D-aspartate, as well as about the functions in which both D-amino acids are involved. These two $\mathrm{D}$-amino acids are the most abundant $\mathrm{D}$-amino acids in mammals [3]. However, the distribution of D-glutamate in the mammalian CNS and the functions in which this D-amino acid is involved have been less studied. It is important to apply immunohistochemistry (IHC) to investigate the tissue distribution of D-amino acids, because this technique shows which cells contain such substances and where D-amino acids are located inside the cells (cell body, dendrites, nerve fibers). IHC allows the determination of the neuroanatomical distribution of $\mathrm{D}$-amino acids in order to gain insight into the possible roles that these substances play in the areas of the CNS in which they are located. To achieve these goals, highly specific antisera against $\mathrm{D}$-amino acids must be obtained. Thus, in this review, we update the information about the anatomical localization of D-glutamate in the mammalian CNS and possible physiological roles played by this $\mathrm{D}$-amino acid. The distribution of D-glutamate is compared with the distribution of both D-serine and D-aspartate and, finally, the development of new tools (highly specific antisera against D-amino acids) for the study of these substances is also reported.

\section{How to obtain highly specific antibodies directed against $\mathrm{D}$-amino acids?}

D-glutamate is a small molecule with a molecular weight below $1 \mathrm{kDa}$ (MW 147.13). Small molecules are not immunogenic, so they are usually linked through a coupling agent (e.g., aldehydes, carbodiimides, picric acid) to a carrier protein [46, 47], since the immune response is triggered by molecules with a mass 1 to $2 \mathrm{kDa}$, which is the minimum size for stimulating antibody production. Animals receiving the immunogen (D-glutamate-coupling agent-carrier protein) will generate different types of polyclonal antibodies, some of which will recognize the conjugated D-glutamate, while other populations will recognize the carrier protein or the coupling agent. Antibodies directed against the carrier protein and the coupling agent could give a spurious signal that must be blocked, reduced or suppressed by later purification and checked after application of the respective controls.

The choice of the coupling agent is very important in order to maintain the original structure and conformation of D-glutamate and hence it should be ensured that the D-glutamate coupled to the carrier protein is identical to that found in the tissue. When glutaraldehyde is used as the coupling agent, a reducing agent such as sodium borohydride, $\mathrm{NaBH}_{4}$, is necessary to reduce the imine groups. Moreover, this agent transforms the double bonds generated during the linkage process into saturated (single) bonds, providing the molecule (e.g., D-glutamate) with a spatial conformation that is generally identical to that of the molecule in vivo. Thus, $\mathrm{NaBH}_{4}$ enables saturation of the imine double bonds [46]. This is very important in such studies that use IHC in which the antibodies must recognize a specific target. This protocol is readily applicable both at the in vitro and at tissue level [8, 44, 46]. In vitro: the antigen used for the immunization of the animals is reduced with $\mathrm{NaBH}_{4}$ (the molecules used for the immunization must be the same as those that will be assayed later in vitro or in the tissue); hence, the antigen used to characterize the antibody affinity and specificity sites were evaluated using the Enzyme Linked Immunosorbent Assay (ELISA) test. In tissue: after perfusion of the animals and processing of the tissue, the slices are reduced with $\mathrm{NaBH}_{4}$ to localize the molecule in its original conformation in the tissue. This process is also easy to use in tissues fixed with glutaraldehyde (immunocytochemical techniques): the background is reduced since the immunogen used and the molecules located in the tissue have the same conformation [44, 46]. Thus, the reduction of the sections with $\mathrm{NaBH}_{4}$ increases the specificity of the immunocytochemical reaction $[44,48]$, allows a spatial change of the antigen, and enables the antibody site to better recognize the three-dimensional structure of the antigen due to a change in the molecular orbital from Sp2 to Sp3 [46]. The tissue treatment described here should be always carried out when D-glutamate has been linked via glutaraldehyde to a carrier protein $[44,46]$. According to the above, it must be confirmed that the antigen (e.g., D-amino acid), that will be used for the immunization of the animals, was not modified during the linkage 
procedure [46]. This is important, for ethical (in order to use a lower number of animals) and technical reasons (to avoid generation of unspecific antibodies generated by an incorrect linkage or a modification of the targeted molecule) [46]. Therefore, the chemical structure of the antigen used for immunization is usually controlled by spectrophotometry (ultraviolet and/or infrared) [44, 46].

The development of new and highly specific antibodies directed against $\mathrm{D}$-amino acids has increased the knowledge on the field (e.g., D-glutamate, D-serine, D-aspartate) according to the protocol carried out by our group [44, 46]. D-glutamate was dissolved in acetate buffer and a second solution was prepared containing bovine serum albumin (BSA), also dissolved in the same buffer (Fig. 1). To conjugate the D-glutamate with BSA, a glutaraldehyde solution was added to the first solution (containing dissolved D-glutamate). The coupling reaction produced yellow color and the addition of $\mathrm{NaBH}_{4}$ rendered the solution translucent (Fig. 1). The $\mathrm{NaBH}_{4}$ saturated the double bonds, and after the mixture became totally translucent, the saturation reaction was over. This final solution was dialyzed against distilled water. Then, rabbits were immunized every two or three weeks over two months (Fig. 1). Each subcutaneous administration was a mixture of a fresh immunogenic conjugate (reduced D-glutamate-glutaraldehyde-BSA) and of complete or incomplete Freund adjuvant, depending on whether it was the first immunization or consecutive ones. After the second immunization, serum samples were obtained. Antisera were preabsorbed with reduced and lyophilized BSA-glutaraldehyde in order to remove antibodies directed against the conjugated carrier protein and neutralize possible spurious signals in later applications such as ELISA or IHC. After this process, the antisera must be centrifuged and the floating phase must be purified. Antisera must be tested before and after the purification process in order to evaluate possible alterations that might occur during the purification process, and no changes in the signal or specificity of the antibodies must be found as a consequence of the pre-purification process.

ELISA tests are used to evaluate the antibodies present in the antisera. In order to obtain an adequate characterization, it is crucial to get the titration values before to carry out the competition experiments (Fig. 1). This is done at $492 \mathrm{~nm}$ and the best dilution is obtained when the optical density is around 1 $[44,47]$. For example, in the case of D-glutamate, several optical densities are obtained for several concentrations of the antibody (dilution 1/40,000: the optical density was 0.5 ; dilution $1 / 20,000$ : 1 ; dilution
1/10,000: 2; dilution 1/5,000: 3 ). The $\mathrm{IC}_{50}$ is the point to be reached and this occurs when there is enough competitor to decrease the optical density to 0.5 . This value is important because it will be used to obtain the avidity of the antibody for a specific target (e.g. D-glutamate) (Fig. 1). In order to obtain the affinity of the antibodies directed against D-amino acids, several solutions containing an identical concentration of the antiserum (e.g. D-glutamate at 1/10,000) are tested using several concentrations of the antigen (e.g. conjugated D-glutamate at $10^{-3} \mathrm{M}, 10^{-4} \mathrm{M}$ etc.). The avidity of the antibody is high when, at a low concentration of the antigen (lower than $10^{-7} \mathrm{M}$ ), the optical density (antibody signal) disappears (Fig. 1). By the use of different antigens the specificity of the antibody is determined (Fig. 1, Table 1). Small differences in chemical structures (e.g., different spatial conformations of the same molecule, chemical groups, and/or chain lengths) are required to determinate the specificity of the antibody (see Table 1 and 2).

The estimated antibody avidity $\left(\mathrm{IC}_{50}\right)$ is fairly high $\left(10^{-8} \mathrm{M}\right.$ for anti-conjugated $\mathrm{D}$-glutamate and $10^{-11} \mathrm{M}$ for anti-conjugated $\mathrm{D}$-serine and $\mathrm{D}$-aspartate antibodies). Moreover, the specificity of the three anti-conjugated $\mathrm{D}$-amino acids antibodies was very high, because the antisera discriminated very well closely conjugated structures. For D-glutamate, the competitors tested were the following: L enantiomer of glutamate, both enantiomers of glutamine and aspartate, D-cysteine, D-methionine, D-tryptophan, D-tyrosine, taurine and GABA (Table 1). For D-serine: L enantiomer of serine, both enantiomers of $D$-aspartate, D-cysteine, D-alanine, L-glutamate, L-glutamine, GABA, glycine, and taurine (see Table 1) and for D-aspartate: L enantiomer of aspartate, both enantiomers of glutamate, and N-methyl-D-aspartate (see Table 2). No cross-reactivity was observed with the molecules studied. An important fact is that anti-conjugated D-glutamate antibodies do not recognize the L enantiomer (L-glutamate) or D-glutamine, which is known to be synthesized in glial cells from D-glutamate [49]. All the conjugate coupled by a glutaraldehyde bond was reduced. This means that no spurious immunoreactivity could be considered, since the antiserum is preabsorbed with reduced glutaraldehyde-BSA before application of ELISA and/or immunocytochemical techniques and that the antibody is specific to the reduced conjugated $\mathrm{D}$-glutamate. That is, the conjugated $\mathrm{D}$-amino acids studied were reduced and were identical to those conjugated molecules used for the immunization of the animals. In summary, the antisera recognized specifically the targeted D-amino acids, since antibodies did not present cross-reactivity with analogous close 


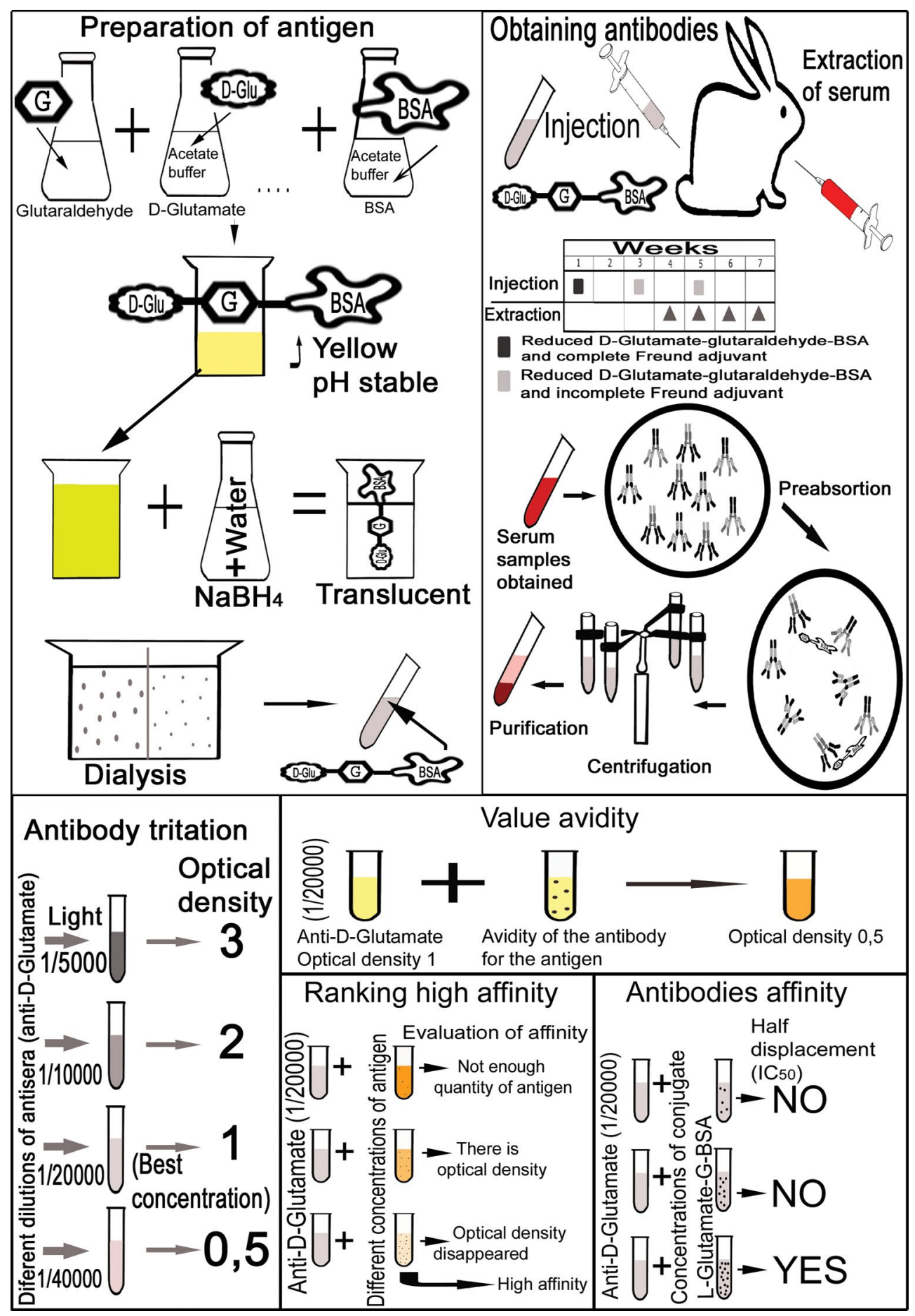

Figure 1. The procedure of developing highly specific antibodies against D-glutamate (see text).

structures, even when comparing $\mathrm{D} / \mathrm{L}$ enantiomers (Table 1 and 2).

\section{Neuroanatomical distribution of D-amino acids}

Using an HPLC technique, the presence of free D-glutamate in the rat brain was first reported in 1995 [38].
Those authors also demonstrated for the first time the presence of free D-glutamate in rat liver and kidney and that the treatment of tissue extracts with D-aspartate oxidase mostly abolished the HPLC peaks of D-glutamate. The same authors also reported that the content of free D-glutamate was lower in females than in males [38]. However, before using highly specific antibodies directed against D-glutamate developed 
Table 1. Affinity and specificity of antibodies directed against conjugated D-glutamate and D-serine

\begin{tabular}{|l|c|}
\hline Compound & $\begin{array}{c}\text { Cross reactivity at } \\
\text { half-displacement }\left(\mathbf{I C}_{\mathbf{5 0}}\right)\end{array}$ \\
\hline D-Glutamate competitors & 1 \\
D-Glutamate-G-BSA & $1 / 50,000$ \\
L-Glutamate-G-BSA & $1 / 50,000$ \\
*Glutamine-G-BSA & $1 / 50,000$ \\
*Aspartate-G-BSA & $1 / 50,000$ \\
D-Cysteine-G-BSA & $1 / 50,000$ \\
D-Methionine-G-BSA & $1 / 50,000$ \\
D-Tryptophan-G-BSA & $1 / 50,000$ \\
D-Tyrosine-G-BSA & $1 / 50,000$ \\
Taurine-G-BSA & $1 / 50,000$ \\
GABA-G-BSA & \\
D-Serine competitors & \\
D-Serine-G-BSA & 1 \\
L-Serine-G-BSA & $1 / 10,000$ \\
D-Cysteine-G-BSA & $1 / 50,000$ \\
D-Alanine-G-BSA & $1 / 50,000$ \\
L-Glutamic acid-G-BSA & $1 / 50,000$ \\
L-Glutamine-G-BSA & $1 / 50,000$ \\
*Aspartate-G-BSA & $1 / 50,000$ \\
GABA-G-BSA & $1 / 50,000$ \\
Glycine-G-BSA & $1 / 50,000$ \\
Taurine-G-BSA & $1 / 50,000$ \\
BSA & $1 / 50,000$ \\
& \\
\hline
\end{tabular}

Using competition ELISA assays, cross-reactivity was calculated from the displacement curves at half-displacement: the best recognized conjugates were D-glutamate-G-BSA and D-Serine-G-BSA, respectively, whose concentration was divided by the concentration of each of the other conjugates. $\mathrm{G}$ - glutaraldehyde. ${ }^{*}$ Means that L and D enantiomers were tested and the results were identical.

by GEMAC S.A. (Saint Jean d'Illac, France), the mapping of immunoreactive structures containing this D-amino acid was not carried out in the mammalian brain until 2007 [44]. In that study, only perikarya/ /dendrites containing D-glutamate were seen in the rat brain, since no D-glutamate-immunoreactive fibers were found. Immunoreactivity was confined to neuronal structures, whereas non-neuronal cells were devoid of immunoreactivity. The distribution of the D-glutamate-immunoreactive cell bodies was very restricted, since these cell bodies were only found in rat mesencephalon and thalamus in the following nuclei/regions: the nucleus of Darkschewitsch, the dorsal and ventral parts of the mesencephalic central grey, the medial habenular nucleus, the superior colliculus, the dorsal raphe nucleus (Fig. 2A-D), the posterior thalamic nuclear group, the subparafascicular thalamic nucleus and above the rostral linear nucleus of the raphe and the posterior commissure (Table 3). Moreover, a cluster of D-glutamate-immunoreactive cell bodies was located below
Table 2. Affinity and specificity of antibodies directed against conjugated D-aspartate

\begin{tabular}{|l|c|}
\hline Compound & $\begin{array}{c}\text { Cross-reactivity } \\
\text { at half-displacement }\left(\mathbf{I C}_{\mathbf{5 0}} \mathbf{)}\right.\end{array}$ \\
\hline D-Aspartate-G-BSA & 1 \\
\hline L-Aspartate-G-BSA & $1 / 10,000$ \\
\hline N-Methyl-D-Aspartate & $1 / 50,000$ \\
\hline *Glutamic acid-G-BSA & $1 / 50,000$ \\
\hline BSA & $1 / 50,000$ \\
\hline
\end{tabular}

Using competition ELISA assays, cross-reactivity was calculated from the displacement curves at half-displacement. The best recognized conjugate was D-Aspartate-G-BSA, whose concentration was divided by the concentration of each of the other conjugates. $\mathrm{G}$ - glutaraldehyde. *Means that $\mathrm{L}$ and $\mathrm{D}$ enantiomers were tested and the results were identical.

the medial forebrain bundle. In general, cell bodies containing D-glutamate were small (10-14 $\mu \mathrm{m}$ in diameter), round or fusiform, and showed none to three short-medium-length dendrites [44]. It should be noted that the distribution and number of such immunoreactive cell bodies differed, depending on the protocol followed for the immunocytochemical technique. In that study [44], two different protocols for IHC were followed. In the first, the sections were reduced with a solution of sodium borohydride prior to carrying out the immunocytochemical protocol [48], whereas in the second protocol the reduction procedure was not carried out. Applying the first method, antibodies recognized the three-dimensional structure of the antigen (D-glutamate) better [46, 48]. When the sections were reduced with sodium borohydride, more nuclei in the central nervous system of the rat showed immunoreactivity (that is, the distribution was more widespread than when such sections were not reduced) and, in addition, a higher number of immunoreactive perikarya containing D-glutamate was observed, in the same nucleus, in $\mathrm{NaBH}_{4}$-reduced sections than in unreduced ones [44].

In the immunohistochemical studies, in order to confirm the specificity of the immunoreactivity it is very important to carry out adequate histological controls. The specificity of the immunocytochemical techniques used is not absolute, and the validity of the results should always be checked with other techniques. The affinity and specificity of antibodies directed against $\mathrm{D}$-amino acids must be tested using ELISA tests and they must also be studied under the same conditions with different competitors in each particular case.

The immunoreactivity for D-glutamate was always confined to neuronal structures (dendrites and cell bodies), but the presence of other D-amino acids 

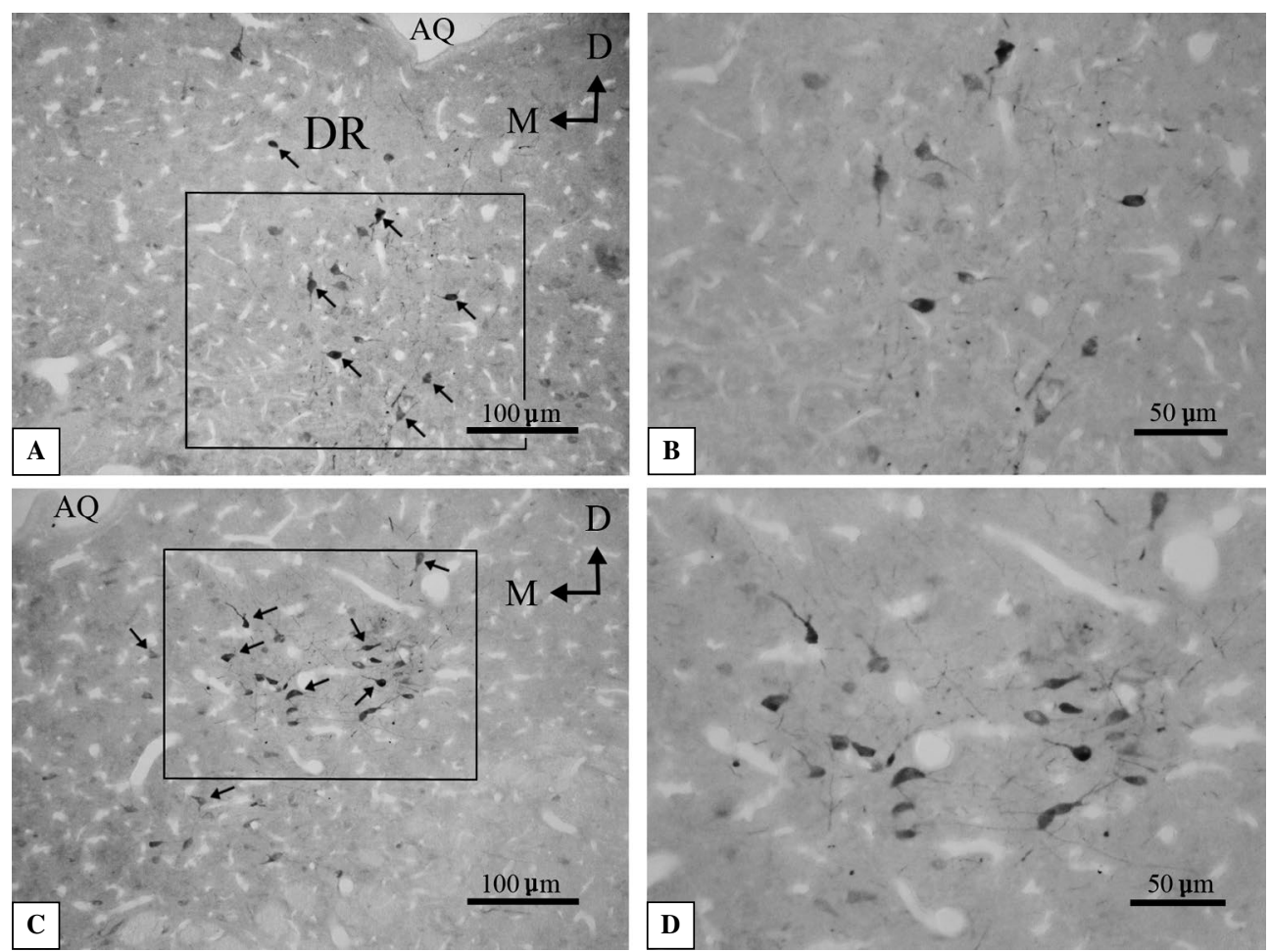

Figure 2. D-glutamate-immunoreactive cell bodies in the rat dorsal raphe nucleus (DR) (A-D). B. High-power magnification of cell bodies containing D-glutamate located in the region demarcated in Figure 2A. D. High-power magnification of the region demarcated in Figure 2C. The anti-conjugated D-glutamate-BSA (reference: AP030) was provided by GEMAC S.A. (France) (see Table 1). Abbreviations: AQ - aqueduct; D - dorsal; M - medial.

such as D-serine has been found in neurons (axons, dendrites and cell bodies) and glial cells [8, 40, 41]. The differences regarding the presence of various D-amino acids inside neurons are unknown, although the location of D-glutamate exclusively in dendrites and cell bodies could be due to the lack of axonal transport mechanisms of this D-amino acid, as it is known to occur for some neuroactive substances (e.g., somatostatin) [50]. Moreover, the distribution of D-glutamate- and D-serine-immunoreactive cell bodies in the mammalian brain (Table 3 ) seems to be quite different, since cell bodies containing D-serine have been located in the cerebral cortex, hippocampus, olfactory bulb, vestibular nuclei, inferior colliculus, motor trigeminal nucleus, trapezoid body, dorsolateral geniculate nucleus (Fig. 3A-D) and in the superior olivary complex $[8,40,41]$. In those regions, no cell bodies containing D-glutamate were observed [44] and, by contrast, in those regions in which cell bodies containing D-glutamate were observed (e.g., dorsal raphe nucleus, mesencephalic central grey, superior colliculus, nucleus of Darkschewitsch, medial habenular nucleus), no cell bodies containing D-serine were found. The presence of fibers containing D-serine has been observed, for example, in the medial and lateral ventroposterior thalamic nuclei (Fig. 3E), the dorsolateral geniculate nucleus, and in the medial geniculate nucleus. D-serine immunoreactive perikarya were small- or medium-sized, round, pyramidal or fusiform, whereas unbranched and smooth D-serine-immunoreactive fibers were short- or medium-sized in length. After the preabsorption of the anti-conjugated-D-serine antibody with D-serine-glutaraldehyde-BSA, the immunoreactivity disappeared (Fig. 3F). No immunoreactive cell bodies containing D-glutamate [44] have been described in any of the above-mentioned thalamic nuclei containing D-serine (Table 3).

D-aspartate-immunoreactive neurons (smallor medium-sized, round or fusiform) were found in the rat brain in the interpeduncular nucleus, the inferior olive, the cochlear nuclei, the hypoglossal nucleus, the facial nucleus, the cerebellum, the paraventricular hypothalamic nucleus, the supraoptic nucleus (Fig. 4A, B), the medial habenular nucleus (Fig. 5A-C), the olfactory bulb, hippocampus and in the septal nuclei [51] (Table 3). This distribution is quite different from that of cell bodies containing D-glutamate [44] or D-serine [8, 40, 41]. Thus, the distribution of 
Table 3. Neuroanatomical distribution of D-glutamate-, D-serine- and D-aspartate-immunoreactive cell bodies in the rat brain

\begin{tabular}{|c|c|c|c|}
\hline & D-glutamate & D-serine & D-aspartate \\
\hline Cerebellum & - & - & + \\
\hline Cerebral cortex & - & + & - \\
\hline Cochlear nuclei & - & - & + \\
\hline Dorsal raphe nucleus & + & - & - \\
\hline Dorsolateral geniculate nucleus & - & + & - \\
\hline Facial nucleus & - & - & + \\
\hline Hippocampus & - & + & + \\
\hline Hypoglossal nucleus & - & - & + \\
\hline Inferior colliculus & - & + & - \\
\hline Inferior olive & - & - & + \\
\hline Interpeduncular nucleus & - & - & + \\
\hline Lateral ventroposterior thalamic nucleus & - & + & - \\
\hline Medial geniculate nucleus & - & + & - \\
\hline Medial habenular nucleus & + & - & + \\
\hline Medial ventroposterior thalamic nucleus & - & + & - \\
\hline Mesencephalic central gray & + & - & - \\
\hline Motor trigeminal nucleus & - & + & - \\
\hline Nucleus of Darkschewitsch & + & - & - \\
\hline Olfactory bulb & - & + & + \\
\hline Paraventricular hypothalamic nucleus & - & - & + \\
\hline Posterior thalamic nuclear group & + & + & - \\
\hline Septal nuclei & - & - & + \\
\hline Subparafascicular nucleus & + & - & - \\
\hline Superior colliculus & + & - & - \\
\hline Superior olivary complex & - & + & - \\
\hline Supraoptic nucleus & - & - & + \\
\hline Trapezoid body & - & + & - \\
\hline Vestibular nuclei & - & + & - \\
\hline
\end{tabular}

The table summarizes data presented in the text and Figures 2-5.

the neuronal populations containing D-glutamate, $\mathrm{D}$-serine or $\mathrm{D}$-aspartate is different in the rat brain (Table 3). Moreover, in comparison with the distribution of the immunoreactive structures containing either $\mathrm{D}$-serine or $\mathrm{D}$-aspartate in the rat brainstem, the distribution of D-glutamate is less widespread. It should be noted that cell bodies containing D-aspartate were described in the medial habenula [51], and in this nucleus the presence of D-glutamate-immunoreactive cell bodies has been also reported [44] (Table 3).This means that a possible coexistence of both D-amino acids could occur in this thalamic nucleus. This could be demonstrated in future studies.

\section{Functional roles of D-amino acids and future studies}

D-serine and D-aspartate play important roles in the mammalian CNS system (e.g., neuronal migration, synaptic plasticity, NMDA receptor-mediated neurotransmission, neurotoxicity, inhibition of melatonin synthesis, pain, brain aging, stimulation of oxytocin synthesis, regulation of steroid synthesis) and they are also involved in brain disorders (e.g., schizophrenia, epilepsy, amyotrophic lateral sclerosis, Alzheimer's disease) [1-3, 8, 9, 11-13, 15, 18-20, 22-24, 26-28, 52]. 


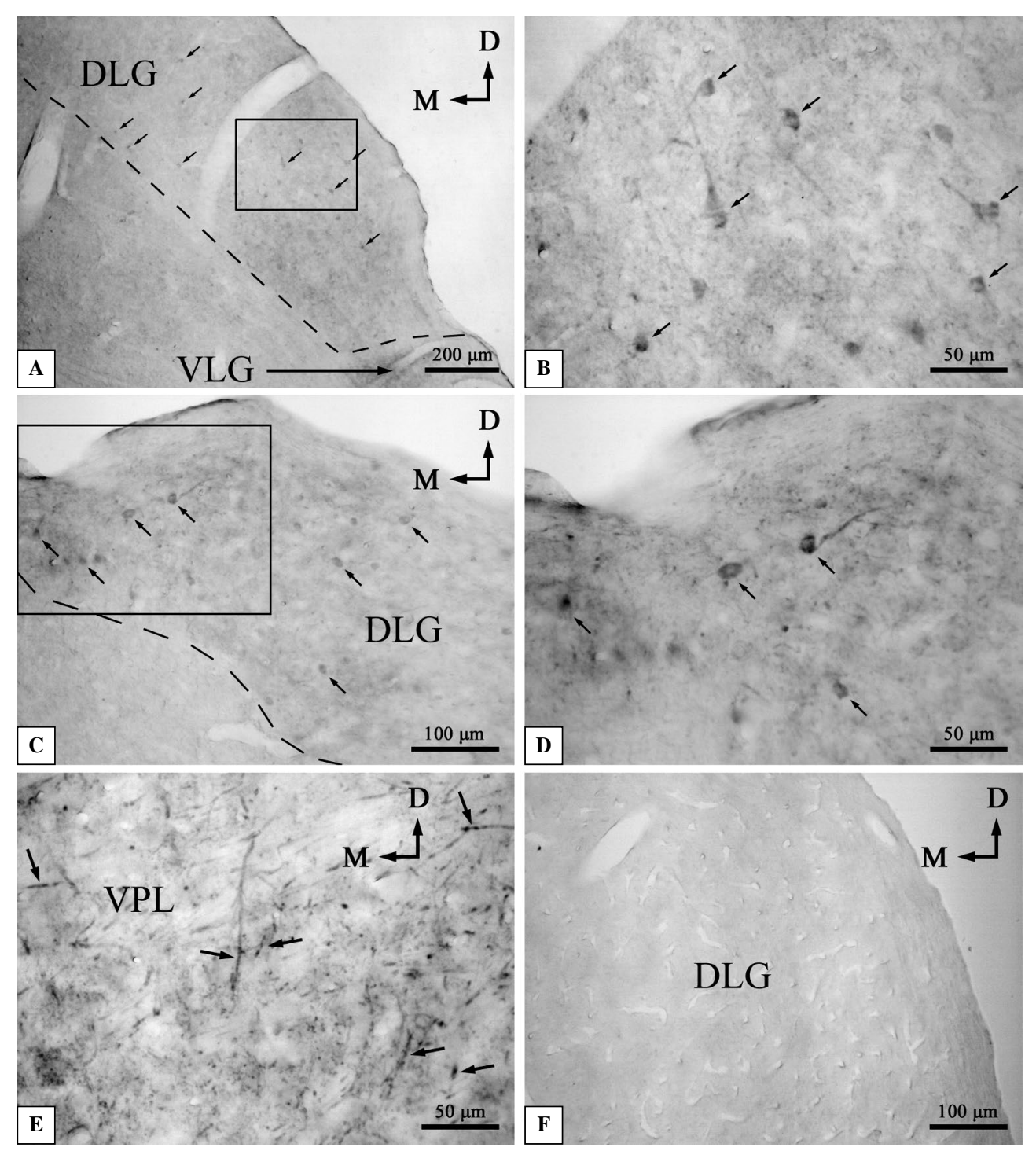

Figure 3. D-serine-immunoreactive cell bodies in the rat dorsolateral geniculate nucleus (A-D). B. High-power magnification of cell bodies containing D-serine located in the region demarcated in Figure 3A. D. High-power magnification of the region demarcated in Figure 3C. E. D-serine-immunoreactive nerve fibers in the lateral ventro-posterior thalamic nucleus. F. Low-power magnification of the dorsolateral geniculate nucleus after preabsorption of the first antibody. (The preabsorption of the first antibody with the corresponding antigen: if the signal is avoided, it demonstrates that there's no background or spurious signal and that signal visualized corresponds to specific signal of the first antibody, means specificity). Note the absence of immunoreactive cell bodies. Arrows indicate immunoreactive cell bodies. Abbreviations: D, dorsal; DLG, dorsolateral geniculate nucleus; M, medial; VLG, ventrolateral geniculate nucleus; VPL, lateral ventroposterior thalamic nucleus. This Figure is published with the permission of GEMAC S.A. Laboratories. The anti-conjugated D-serine-BSA (reference: AP041) was provided by GEMAC S.A. (France) (see Table 1).

The neuroanatomical data reported above indicate that D-glutamate has a very restricted anatomical distribution in the rat brain; although the possibility that the immunohistochemical techniques used might be insufficiently sensitive to visualize all the profiles containing D-glutamate in the brain cannot be ruled out. It is possible that other neuronal structures might contain low levels of D-glutamate, undetectable with the technical approach used. The restricted distribu- tion of D-glutamate suggests that this D-amino acid could be involved in very specific actions that will have to be addressed in the future. However, according to the anatomical distribution reported for D-glutamate in the rat brain, this D-amino acid could be involved for example in auditive, visual and analgesic mechanisms, since D-glutamate-immunoreactive cell bodies were observed in the superior colliculus and in the mesencephalic central grey [44]. The presence of 

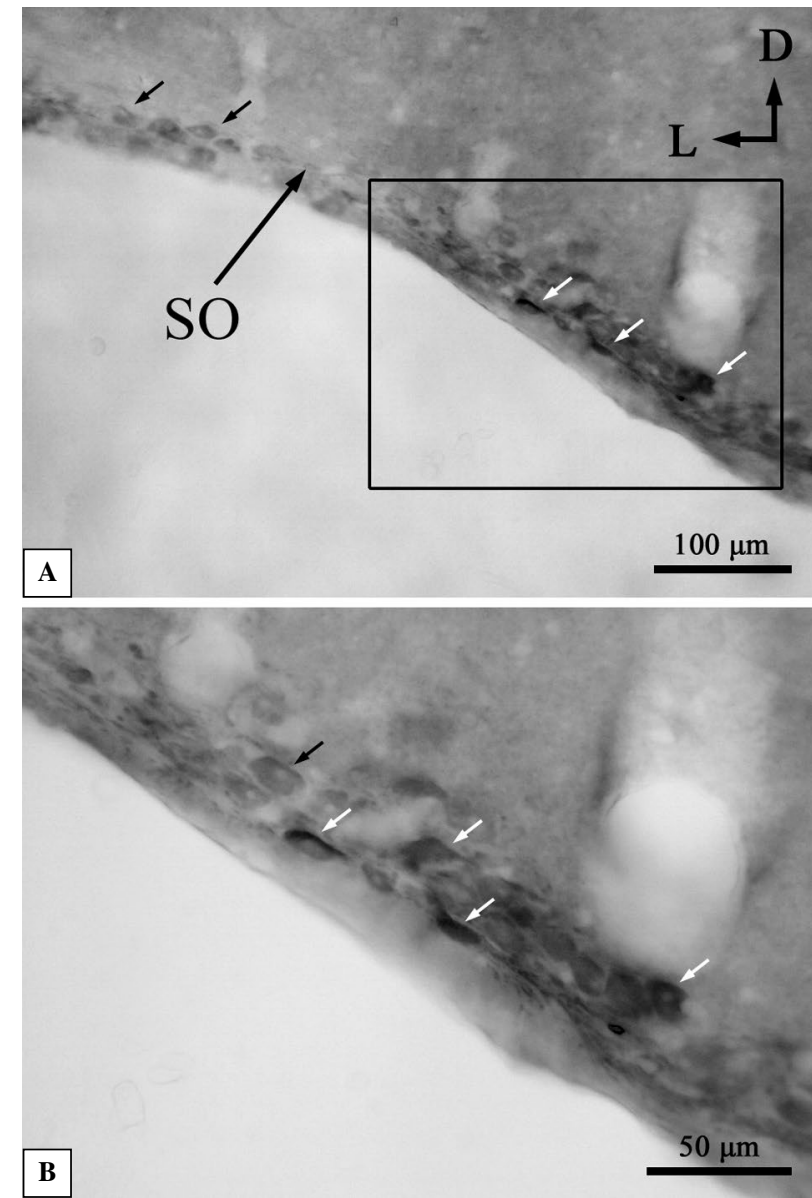

Figure 4. D-aspartate-immunoreactive cell bodies in the supraoptic nucleus (A, B). B. High-power magnification of cell bodies containing D-aspartate located in the region demarcated in Figure 4A. Arrows indicate immunoreactive cell bodies. Abbreviations: D - dorsal; L - lateral; SO - supraoptic nucleus. This Figure is published with the permission of GEMAC S.A. Laboratories. The anti-conjugated D-aspartate-BSA (reference: AP024) used was provided by GEMAC S.A. (France) (see Table 2).

D-glutamate in the medial habenula nucleus suggests that this D-amino acid could be involved in learning, memory, attention, sleep/wake cycles and anxiety [53-55]. Moreover, D-glutamate could be involved in other functions in the rat brain nuclei in which cell bodies containing this D-amino acid were observed. It was demonstrated that D-glutamate is a weak, although effective, competitor for a L-glutamate transporter cloned from glial cells [49], an effective substrate for a low-affinity glutamate uptake system [49] and is taken up into glial cells and converted into D-glutamine [49], this substance being exported from glial cells and taken up by neurons [30]. Moreover, D-glutamate was shown to be a potent natural inhibitor of glutathione synthesis [49] and a low affinity
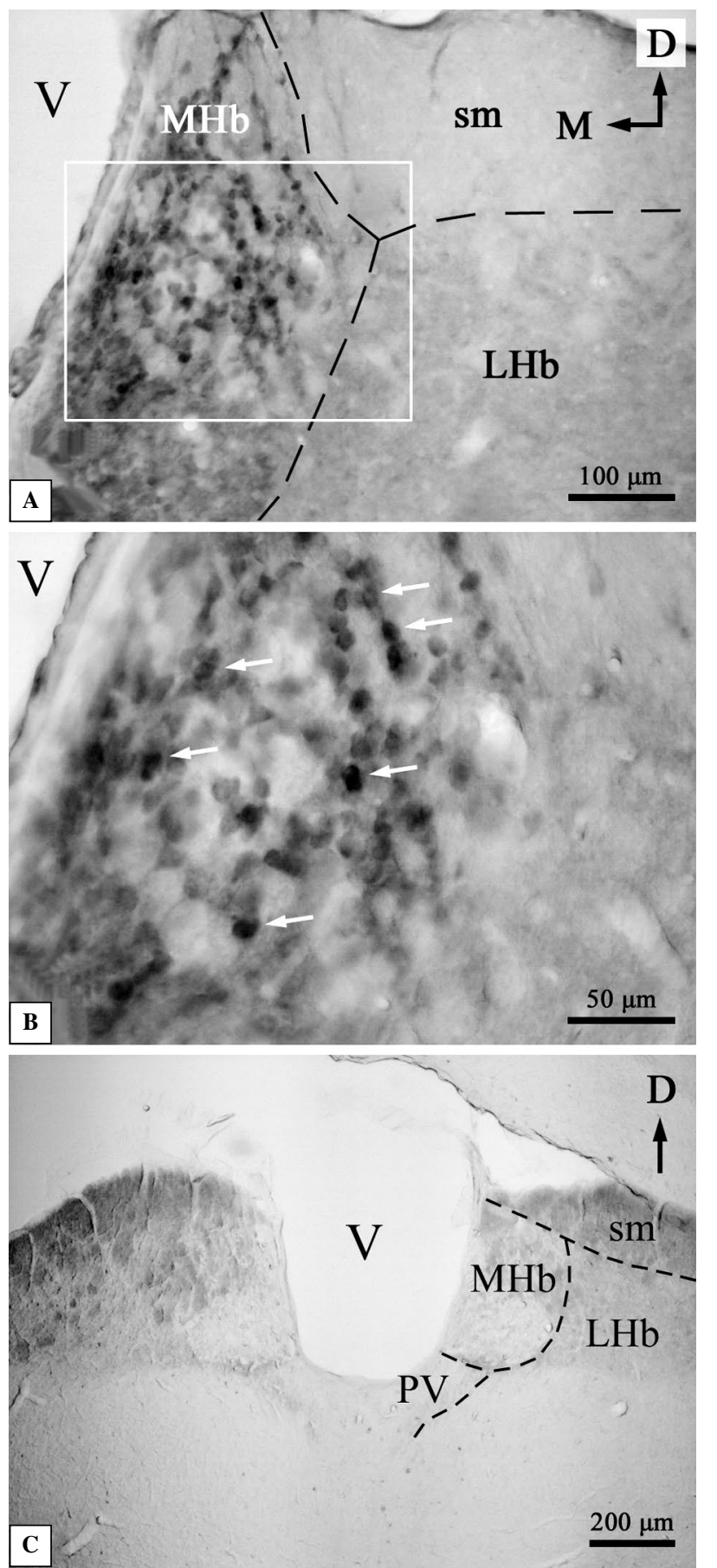

Figure 5. D-aspartate-immunoreactive cell bodies in the medial habenula (A, B). B. High-power magnification of cell bodies containing $\mathrm{D}$-aspartate located in the region demarcated in Figure 5A. C. Low-power magnification of the habenular complex after preabsorption of the first antibody. Note the absence of immunoreactive cell bodies. Arrows indicate immunoreactive cell bodies. Abbreviations: D - dorsal; LHb - lateral habenula; M - medial; MHb — medial habenula; PV — paraventricular thalamic nucleus; sm - stria medullaris thalamus; $\mathrm{V}$ - ventricle. This Figure is published with the permission of GEMAC S.A. Laboratories. The anti-conjugated D-aspartate-BSA (reference: AP024) used was provided by GEMAC S.A. (France) (see Table 2). 
NMDA receptor agonist [30]. These data suggest that the physiological role played by D-glutamate present in the rat mesencephalic and thalamic neurons should be addressed in future studies.

An accumulation of D-glutamate in neurons located in the mesencephalon and thalamus of the rat has also been reported, suggesting that these neurons have a mechanism for the uptake of D-glutamate [44]. Thus, incubation of rat brain slices with D-glutamate increased D-glutamate immunoreactivity in comparison with the immunoreactivity observed in perfused fixed rat brain sections (not incubated with D-glutamate) and in comparison with the results (no immunoreactivity) found in rat brain sections not incubated with D-glutamate (brain slice experiments) [44]. It should be noted that when rat brain slices were treated with D-glutamate (brain slice experiments), immunoreactive cell bodies were observed and these cell bodies showed the same distribution in the rat central nervous system when compared with the immunoreactivity seen in perfused fixed rat brain sections (not incubated with D-glutamate). This suggests that the D-glutamate present in cell bodies could act as a false neurotransmitter that is exported to the extracellular space and that could be taken up by neurons [44]. Other authors have also suggested that $\mathrm{D}$-glutamate is released as a false transmitter [30], although the absence of D-glutamate in nerve fibers and terminals argues against a possible transmitter role of this D-amino acid. Thus, the action of D-glutamate as a neurotransmitter is a point that will have to be addressed in the future.

The neuroanatomical distribution of D-glutamate in the rat brain suggests that it may play hitherto unsuspected roles and hence be a candidate for novel physiologically active substances. Future neuroanatomical and physiological studies should be carried out to collect more data and gain further insight into the functional roles of D-glutamate in the rat brain. Such studies should attempt to unravel whether D-glutamate acts as a neuromodulator/neurotransmitter. Moreover, it would be very interesting to study the distribution of D-glutamate and other $\mathrm{D}$-amino acids in the human/monkey brain using new, highly specific antisera directed against them.

To sum up, our knowledge of D-glutamate function in the mammalian CNS is still quite limited. The advent of high-affinity and high-specificity antisera directed against each conjugated D-amino acid, whose discrimination between the L- and D- enantiomers, as well as other molecules, guarantees specific recognition of a given D-amino acid. By using immunohistochemical techniques D-amino acids can be readily visualized, and such approach will increase our understanding of D-amino acids function of in the mammalian CNS.

\section{Acknowledgements}

This work has been supported by GEMAC S.A. Laboratories (Saint Jean d'Illac, France) and IDRPHT (Talence, France). The authors wish to thank Mr. N. Skinner (University of Salamanca, Spain) for supervising the English text.

\section{Conflicts of interest}

M. Geffard, M. Husson and A. Mangas are ascribed to the company GEMAC S.A. which partially supported the work. This company was the source of the antibodies used in the studies reviewed.

\section{References}

1. Hamase K, Morikawa A, Zaitsu K. D-Amino acids in mammals and their diagnostic value. J Chromatogr B Analyt Technol Biomed Life Sci. 2002; 781(1-2): 73-91, indexed in Pubmed: 12450654.

2. Hamase K, Morikawa A, Etoh S, et al. Analysis of small amounts of D-amino acids and the study of their physiological functions in mammals. Anal Sci. 2009; 25(8): 961-968, indexed in Pubmed: 19667471.

3. Genchi G. An overview on D-amino acids. Amino Acids. 2017; 49(9): 1521-1533, doi: 10.1007/s00726-017-2459-5, indexed in Pubmed: 28681245.

4. Mothet JP, Snyder SH. Brain D-amino acids: a novel class of neuromodulators. Amino Acids. 2012; 43(5): 1809-1810, doi: 10.1007/s00726-012-1403-y, indexed in Pubmed: 22986446.

5. Ohide H, Miyoshi Y, Maruyama R, et al. D-Amino acid metabolism in mammals: biosynthesis, degradation and analytical aspects of the metabolic study. J Chromatogr B Analyt Technol Biomed Life Sci. 2011; 879(29): 3162-3168, doi: 10.1016/j. jchromb.2011.06.028, indexed in Pubmed: 21757409.

6. Kiriyama Y, Nochi H. D-amino acids in the nervous and endocrine systems. Scientifica (Cairo). 2016; 2016: 6494621, doi: 10.1155/2016/6494621, indexed in Pubmed: 28053803.

7. Lin CH, Yang HT, Chiu CC, et al. Blood levels of D-amino acid oxidase vs. D-amino acids in reflecting cognitive aging. Sci Rep. 2017; 7(1): 14849, doi: 10.1038/s41598-017-13951-7, indexed in Pubmed: 29093468.

8. Mothet J-P. Brain derived D-serine: from synthesis to function. In: Mangas A, Coveñas R, Geffard M, eds. Brain Molecules: from Vitamins to Molecules for Axonal Guidance. Trivandrum: Transworld Research Network; 2008: 105-129.

9. Errico F, Napolitano F, Nisticò R, et al. New insights on the role of free D-aspartate in the mammalian brain. Amino Acids. 2012; 43(5): 1861-1871, doi: 10.1007/s00726-012-1356-1, indexed in Pubmed: 22851050.

10. Fuchs SA, Berger R, Klomp LWJ, et al. D-amino acids in the central nervous system in health and disease. Mol Genet Metab. 2005; 85(3): 168-180, doi: 10.1016/j.ymgme.2005.03.003, indexed in Pubmed: 15979028.

11. Gozzi A, Herdon H, Schwarz A, et al. Pharmacological stimulation of NMDA receptors via co-agonist site suppresses fMRI response to phencyclidine in the rat. Psychopharmacology (Berl). 2008; 201(2): 273-284, doi: 10.1007/s00213-008-1271-z, indexed in Pubmed: 18704372. 
12. Tsai G, Yang P, Chung LC, et al. D-serine added to antipsychotics for the treatment of schizophrenia. Biol Psychiatry. 1998; 44(11): 1081-1089, indexed in Pubmed: 9836012.

13. Ryu HJ, Kim JE, Yeo SI, et al. Potential roles of D-serine and serine racemase in experimental temporal lobe epilepsy. J Neurosci Res. 2010; 88(11): 2469-2482, doi: 10.1002 /jnr.22415, indexed in Pubmed: 20623543.

14. Ishiwata $\mathrm{S}$, Umino A, Nishikawa T. Involvement of neuronal and glial activities in control of the extracellular d-serine concentrations by the AMPA glutamate receptor in the mouse medial prefrontal cortex. Neurochem Int. 2017 [Epub ahead of print], doi: 10.1016/j.neuint.2017.09.009, indexed in $\mathrm{Pu}$ bmed: 28966065.

15. Li Z, Xing Y, Guo X, et al. Development of an UPLC-MS/ /MS method for simultaneous quantitation of $11 \mathrm{~d}$-amino acids in different regions of rat brain: Application to a study on the associations of d-amino acid concentration changes and Alzheimer's disease. J Chromatogr B Analyt Technol Biomed Life Sci. 2017; 1058: 40-46, doi: 10.1016/j. jchromb.2017.05.011, indexed in Pubmed: 28531844.

16. Bardaweel SK, Alzweiri M, Ishaqat AA. D-Serine in neurobiology: CNSneurotransmission and neuromodulation. Can J Neurol Sci. 2014; 41(2): 164-176, indexed in Pubmed: 24534026.

17. Xia J, Xiong H. Neuropathogenesis of HIV-1-associated neurocognitive disorders: a possible involvement of D-serine. Int J Physiol Pathophysiol Pharmacol 2013; 5: 137-147. , indexed in Pubmed: 24044033.

18. Paul P, de Belleroche J. The role of D-amino acids in amyotrophic lateral sclerosis pathogenesis: a review. Amino Acids. 2012; 43(5): 1823-1831, doi: 10.1007/s00726-012-1385-9, indexed in Pubmed: 22890612.

19. Billard JM. D-Amino acids in brain neurotransmission and synaptic plasticity. Amino Acids. 2012; 43(5): 1851-1860, doi: 10.1007/s00726-012-1346-3, indexed in Pubmed: 22886346.

20. Takigawa Y, Homma H, Lee JA, et al. D-aspartate uptake into cultured rat pinealocytes and the concomitant effect on L-aspartate levels and melatonin secretion. Biochem Biophys Res Commun. 1998; 248(3): 641-647, doi: 10.1006/bbrc.1998.8971, indexed in Pubmed: 9703979.

21. Neidle A, Dunlop DS. Developmental changes in free D-aspartic acid in the chicken embryo and in the neonatal rat. Life Sci. 1990; 46(21): 1517-1522, indexed in Pubmed: 2355797.

22. Wang $\mathrm{H}$, Wolosker $\mathrm{H}$, Pevsner J, et al. Regulation of rat magnocellular neurosecretory system by $\mathrm{D}$-aspartate: evidence for biological role(s) of a naturally occurring free $\mathrm{D}$-amino acid in mammals. J Endocrinol. 2000; 167(2): 247-252, indexed in Pubmed: 11054638.

23. Palazzo E, Luongo L, Guida F, et al. D-Aspartate drinking solution alleviates pain and cognitive impairment in neuropathic mice. Amino Acids. 2016; 48(7): 1553-1567, doi: 10.1007/s00726-016-2205-4, indexed in Pubmed: 27115160.

24. Errico F, Di Maio A, Marsili V, et al. Bimodal effect of D-aspartate on brain aging processes: insights from animal models. J Biol Regul Homeost Agents. 2013; 27(2 Suppl): 49-59, indexed in Pubmed: 24813315.

25. Punzo D, Errico F, Cristino L, et al. Age-related changes in D-aspartate oxidase promoter methylation control extracellular D-aspartate levels and prevent precocious cell death during brain aging. J Neurosci. 2016; 36(10): 3064-3078, doi: 10.1523/ /JNEUROSCI.3881-15.2016, indexed in Pubmed: 26961959.

26. Santillo A, Pinelli C, Burrone L, et al. Induced synthesis of $\mathrm{P} 450$ aromatase and $17 \beta$-estradiol by D-aspartate in frog brain. J Exp Biol. 2012; 215(Pt 20): 3559-3565, doi: 10.1242/ /jeb.073296, indexed in Pubmed: 22771744.
27. Nuzzo T, Sacchi S, Errico F, et al. Decreased free d-aspartate levels are linked to enhanced d-aspartate oxidase activity in the dorsolateral prefrontal cortex of schizophrenia patients. NPJ Schizophr. 2017; 3: 16, doi: 10.1038/s41537-017-0015-7, indexed in Pubmed: 28560262.

28. de Bartolomeis A, Errico F, Aceto G, et al. D-aspartate dysregulation in $\operatorname{Ddo}(-/-)$ mice modulates phencyclidine-induced gene expression changes of postsynaptic density molecules in cortex and striatum. Prog Neuropsychopharmacol Biol Psychiatry. 2015; 62: 35-43, doi: 10.1016/j.pnpbp.2015.05.003, indexed in Pubmed: 25979765.

29. Raj D, Langford M, Krueger S, et al. Regulatory responses to an oral D-glutamate load: formation of D-pyrrolidone carboxylic acid in humans. Am J Physiol Endocrinol Metab. 2001; 280(2): E214-E220, doi: 10.1152/ajpendo.2001.280.2.E214, indexed in Pubmed: 11158923.

30. Pan ZZ, Tong G, Jahr CE. A false transmitter at excitatory synapses. Neuron. 1993; 11(1): 85-91, indexed in Pubmed: 8101712.

31. Fisher G, Petrucelli L, Gardner C, et al. Freed-amino acids in human cerebrospinal fluid of Alzheimer disease, multiple sclerosis, and healthy control subjects. Mol Chem Neuropathol. 1994; 23(2-3): 115-124, doi: 10.1007/bf02815405.

32. Yoshimura T, Esak N. Amino acid racemases: functions and mechanisms. J Biosci Bioeng. 2003; 96(2): 103-109, indexed in Pubmed: 16233494.

33. Smith SM, Uslaner JM, Hutson PH. The therapeutic potential of D-amino acid oxidase (DAAO) inhibitors. Open Med Chem J. 2010; 4: 3-9, doi: 10.2174/1874104501004020003, indexed in Pubmed: 20648222.

34. Weatherly CA, Du S, Parpia C, et al. D-amino acid levels in perfused mouse brain tissue and blood: a comparative study. ACS Chem Neurosci. 2017; 8(6): 1251-1261, doi: 10.1021/acschemneuro.6b00398, indexed in Pubmed: 28206740.

35. Karakawa S, Shimbo K, Yamada N, et al. Simultaneous analysis of D-alanine, D-aspartic acid, and D-serine using chiral high-performance liquid chromatography-tandem mass spectrometry and its application to the rat plasma and tissues. J Pharm Biomed Anal. 2015; 115: 123-129, doi: 10.1016/j. jpba.2015.05.024, indexed in Pubmed: 26186615.

36. Han H, Miyoshi Y, Koga R, et al. Changes in D-aspartic acid and D-glutamic acid levels in the tissues and physiological fluids of mice with various D-aspartate oxidase activities. J Pharm Biomed Anal. 2015; 116: 47-52, doi: 10.1016/j. jpba.2015.05.013, indexed in Pubmed: 26058797.

37. Miyoshi Y, Koga R, Oyama T, et al. HPLC analysis of naturally occurring free D-amino acids in mammals. J Pharm Biomed Anal. 2012; 69: 42-49, doi: 10.1016/j.jpba.2012.01.041, indexed in Pubmed: 22386210.

38. Kera Y, Aoyama H, Matsumura H, et al. Presence of free $\mathrm{D}$-glutamate and $\mathrm{D}$-aspartate in rat tissues. Biochim $\mathrm{Bi}$ ophys Acta. 1995; 1243(2): 283-286, indexed in Pubmed: 7873575.

39. Song $\mathrm{Y}$, Feng $\mathrm{Y}, \mathrm{Lu} \mathrm{X}$, et al. D-Amino acids in rat brain measured by liquid chromatography/tandem mass spectrometry. Neurosci Lett. 2008; 445(1): 53-57, doi: 10.1016/j.neulet.2008.08.058, indexed in Pubmed: 18775473.

40. Yasuda E, Ma N, Semba R. Immunohistochemical evidences for localization and production of $\mathrm{D}$-serine in some neurons in the rat brain. Neurosci Lett. 2001; 299(1-2): 162-164, indexed in Pubmed: 11166963.

41. Puyal J, Martineau M, Mothet JP, et al. Changes in D-serine levels and localization during postnatal development of the 
rat vestibular nuclei. J Comp Neurol. 2006; 497(4): 610-621, doi: 10.1002/cne.21016, indexed in Pubmed: 16739185.

42. Williams SM, Diaz CM, Macnab LT, et al. Immunocytochemical analysis of $\mathrm{D}$-serine distribution in the mammalian brain reveals novel anatomical compartmentalizations in glia and neurons. Glia. 2006; 53(4): 401-411, doi: 10.1002/glia.20300, indexed in Pubmed: 16342169.

43. Liu YH, Wang L, Wei LC, et al. Up-regulation of D-serine might induce GABAergic neuronal degeneration in the cerebral cortex and hippocampus in the mouse pilocarpine model of epilepsy. Neurochem Res. 2009; 34(7): 1209-1218, doi 10.1007/s11064-008-9897-0, indexed in Pubmed: 19123037.

44. Mangas A, Coveñas R, Bodet D, et al. Immunocytochemical visualization of D-glutamate in the rat brain. Neuroscience. 2007; 144(2): 654-664, doi: 10.1016/j.neuroscience.2006.09.045, indexed in Pubmed: 17084987.

45. Ding X, Ma N, Nagahama M, et al. Localization of D-serine and serine racemase in neurons and neuroglias in mouse brain. Neurol Sci. 2011; 32(2): 263-267, doi: 10.1007/s10072010-0422-2, indexed in Pubmed: 20890627.

46. Mangas A, Coveñas R, Bodet D, et al. Antisera and immunocytochemical techniques. In: Mangas A, Coveñas R, Geffard M, eds. Brain Molecules: from Vitamins to Molecules for Axonal Guidance. Trivandrum: Transworld Research Network: 2008: 1-25.

47. Mangas A, Yajeya J, Gonzalez N, et al. Detection of pantothenic acid-immunoreactive neurons in the rat lateral septal nucleus by a newly developed antibody. Folia Histochem Cytobiol. 2016; 54(4): 186-192, doi: 10.5603/FHC.a2016.0024, indexed in Pubmed: 27966211.

48. Chagnaud JL, Campistron G, Geffard M. Monoclonal antibody directed against glutaraldehyde conjugated glutamate and immunocytochemical applications in the rat brain.
Brain Research. 1989; 481(1): 175-180, doi: 10.1016/00068993(89)90500-3.

49. Pow DV, Crook DK. Direct immunocytochemical evidence for the transfer of glutamine from glial cells to neurons: use of specific antibodies directed against the d-stereoisomers of glutamate and glutamine. Neuroscience. 1996; 70(1): 295-302, indexed in Pubmed: 8848133.

50. de León M, Coveñas R, Narváez JA, et al. Somatostatin-28 (1-12)-like immunoreactivity in the cat diencephalon. Neuropeptides. 1991; 19(2): 107-117, doi: 10.1016/01434179(91)90140-e.

51. Schell MJ, Cooper OB, Snyder SH. D-aspartate localizations imply neuronal and neuroendocrine roles. Proc Natl Acad Sci U S A. 1997; 94(5): 2013-2018, indexed in Pubmed: 9050896.

52. Errico F, Napolitano F, Squillace M, et al. Decreased levels of $\mathrm{D}$-aspartate and NMDA in the prefrontal cortex and striatum of patients with schizophrenia. J Psychiatr Res. 2013; 47(10): 1432-1437, doi: 10.1016/j.jpsychires.2013.06.013, indexed in Pubmed: 23835041.

53. Lecourtier L, Kelly PH. A conductor hidden in the orchestra? Role of the habenular complex in monoamine transmission and cognition. Neurosci Biobehav Rev. 2007; 31(5): 658-672, doi: 10.1016/j.neubiorev.2007.01.004, indexed in Pubmed: 17379307

54. Hikosaka O, Sesack SR, Lecourtier L, et al. Habenula: crossroad between the basal ganglia and the limbic system. J Neurosci. 2008; 28(46): 11825-11829, doi: 10.1523/JNEUROSCI.3463-08.2008, indexed in Pubmed: 19005047.

55. Guilding C, Hughes ATL, Piggins HD. Circadian oscillators in the epithalamus. Neuroscience. 2010; 169(4): 1630-1639, doi: 10.1016/j.neuroscience.2010.06.015, indexed in Pubmed: 20547209 